\title{
Hierarchical rank-based veiling light estimation for underwater dehazing
}

\section{Simon Emberton}

s.emberton@qmul.ac.uk

Lars Chittka

l.chittka@qmul.ac.uk

Andrea Cavallaro

a.cavallaro@qmul.ac.uk
Centre for Intelligent Sensing,

Queen Mary University of London

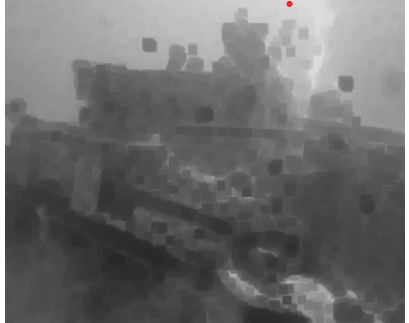

(a)

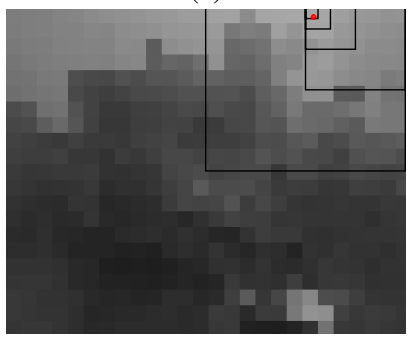

(c)

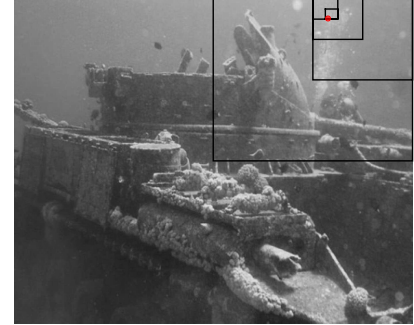

(b)

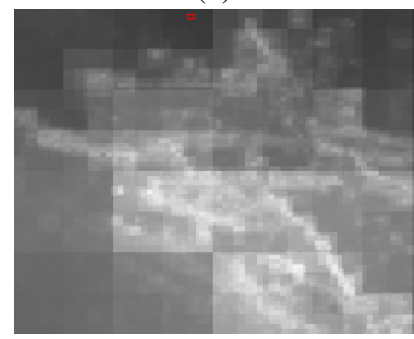

(d)

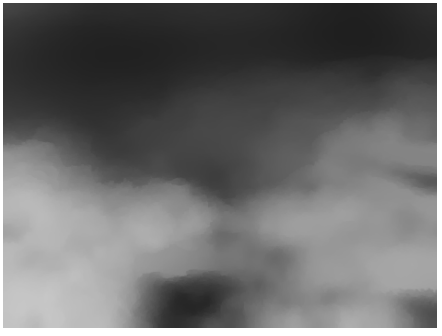

(a)

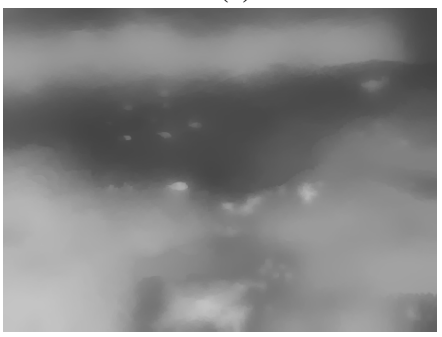

(c)

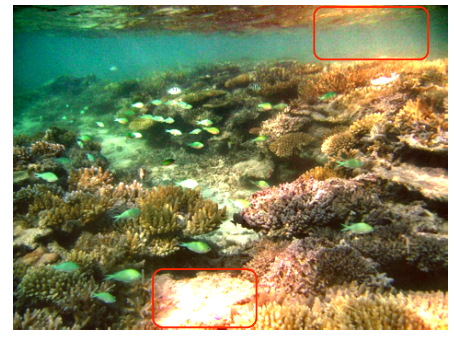

(b)

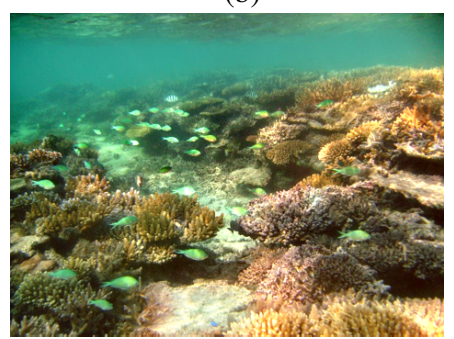

(d)
Figure 1: Comparison of veiling light estimation methods. Veiling light estimates are taken from locations highlighted in red. Current methods are biased toward areas of the Shipwreck image with bright objects (e.g. bubbles) whereas our approach is able to avoid these regions. (a) Underwater Dark Channel Prior [3]. (b) Quad-tree subdivision methods on the input image converted to greyscale [7] and (c) into blocks that are filled with their minimum value [9]. (d) Proposed method.

In this paper we introduce a single image dehazing approach for underwater scenes. We employ a hierarchical rank-based method to estimate the veiling light and an adapted transmission estimation step which prevents oversaturation and artefacts in the dehazed image.

Veiling light is the atmospheric light that is scattered from particles in a hazy scene into the line of sight of an imaging device, contributing to image degradation and resulting in images with low contrast and colour cast [8]. Veiling light estimation is an important step in single image dehazing because it identifies the colour of the atmospheric light which the dehazing process aims to remove. The veiling light can be best estimated from the region of an image that is the most haze-opaque [6]. The transmission is the part of the light that is not scattered by the haze and reaches the camera [8].

Single image dehazing methods improve the visibility of scenes which have been degraded due to scattered light such as fog, haze and smoke by removing the haze in a scene so that objects can be seen more clearly [6]. Dehazing methods can also be used to improve the visibility in underwater images $[1,2,3,5]$.

One of the major challenges for underwater dehazing approaches is to avoid selecting pixels from bright objects during the estimation of the veiling light. We propose a method which aims to avoid objects in the veiling light detection step. We find that regions with objects contain more edges and show more variation within each colour channel than pure veiling light regions. We also consider all regions of an image in the final decision of which region is the most heavily veiled. Fig. 1 compares state-of-the-art veiling light estimation methods together with our proposed approach.

Another challenge is to estimate a transmission map which avoids output dehazed images suffering from oversaturation and artefacts in the background regions. A region-based approach is used to find optimal transmission values for areas that suffer from oversaturation. We also locate background regions through superpixel segmentation and clustering,
Figure 2: Transmission map (a) generated with green-blue dark channel (refined with weighted least squares (WLS) filter [4]) and (b) the resulting dehazed image. Red boxes highlight an area which is oversaturated due to a bright object being underestimated in the transmission map and a background region containing noise and artefacts. (c) Transmission map with adaptations for truncated and background regions (refined with WLS filter) (d) and the proposed resulting dehazed image.

and adapt the transmission values in these regions so to avoid artefacts (Fig. 2).

The dehazed images produced by our method show good performance in comparison to the state of the art across a range of underwater images when evaluated both subjectively and on a number of commonly used quantitative measures.

[1] N. Carlevaris-Bianco, A. Mohan, and R. M. Eustice. Initial results in underwater single image dehazing. In Proc. of IEEE OCEANS, Sept. 2010.

[2] J. Y. Chiang and Y. C Chen. Underwater image enhancement by wavelength compensation and dehazing. IEEE Trans. on Image Processing, 21(4):1756-1769, Apr. 2012.

[3] P. Drews-Jr, E. Nascimento, F. Moraes, S. Botelho, M. Campos, R. Grande-Brazil, and B. Horizonte-Brazil. Transmission estimation in underwater single images. In Proc. of IEEE ICCV, Dec. 2013.

[4] Z. Farbman, R. Fattal, D. Lischinski, and R. Szeliski. Edgepreserving decompositions for multi-scale tone and detail manipulation. ACM Trans. on Graphics, 27(3):67, Aug. 2008.

[5] A. Galdran, D. Pardo, A. Picon, and A. Alvarez-Gila. Automatic red-channel underwater image restoration. Journal of Visual Communication and Image Representation, 26:132-145, Jan. 2015.

[6] K. He, J. Sun, and X. Tang. Single image haze removal using dark channel prior. IEEE Trans. on PAMI, 33(12):2341-2353, Dec. 2011.

[7] J. H. Kim, J. Y. Sim, and C. S. Kim. Single image dehazing based on contrast enhancement. In Proc. of IEEE ICASSP, May 2011.

[8] H. Koschmieder. Theorie der horizontalen Sichtweite: Kontrast und Sichtweite. Keim \& Nemnich, 1925.

[9] D. Park, H. Park, D. K. Han, and H. Ko. Single image dehazing with image entropy and information fidelity. In Proc. of IEEE ICIP, Oct. 2014. 